\title{
VIRTUAL REALITY STORIES FOR CONSTRUCTION TRAINING SCENARIOS: THE CASE OF SOCIAL DISTANCING AT THE CONSTRUCTION SITE
}

\author{
FELIPE MUÑOZ-LA RIVERA ${ }^{1,2,3}$, JAVIER MORA-SERRANO ${ }^{1,2} \&$ EUGENIO OÑATE ${ }^{1,2}$ \\ ${ }^{1}$ International Centre for Numerical Methods in Engineering, Universitat Politècnica de Catalunya, Spain \\ ${ }^{2}$ School of Civil Engineering, Universitat Politècnica de Catalunya, Spain \\ ${ }^{3}$ School of Civil Engineering, Pontificia Universidad Católica de Valparaíso, Chile
}

\begin{abstract}
The architecture, engineering, construction and operation industry has high accident rates, and its workers are susceptible to certain risks due to the variability of their worksites, the tasks they perform and the equipment they use. Of particular interest is the coexistence of workers and heavy machinery in the same space, which is one of the main causes of high accident rates. Increasing digitisation also incorporates the use of new systems (e.g. UAVs, autonomous vehicles, robotic devices), which must function while preserving worker safety. As such, worker training is also essential in these contexts. Research indicates that occupational risk prevention training in construction is not satisfactory. Traditional methods based on lectures (where the student does not take an active role) and traditional test-type assessments do not result in significant learning and fail to create a culture of prevention among workers. In the context of the COVID-19 pandemic, the construction industry has had to incorporate new prevention measures to continue projects while protecting workers' health and safety. Virtual reality has demonstrated advantages in adapting to different contexts, allowing for the agile development of training experiences with immersive characteristics and more significant learning than traditional methods. This research shows the application and agile development of a training experience for social distancing at construction sites to prevent COVID-19 transmission, based on virtual reality and building information modelling and using serious games as a teaching strategy.

Keywords: virtual reality, BIM, construction site, safety in construction, storytelling, job safety analysis technicians (JSAt), COVID-19.
\end{abstract}

\section{INTRODUCTION}

The construction industry is highly relevant to countries' development, accounting for about $6 \%$ of the world's gross domestic product [1]. It is also one of the most dangerous industries worldwide, with more than $30 \%$ of total recorded accidents in the sector being fatal [2]. Construction sites are complex and demonstrate diverse work dynamics, including workers with different training levels, disciplines and activities [3]. In addition, working outside subjects construction workers to unpredictable weather conditions This, coupled with the use of heavy machinery, dangerous tools and large materials, makes it difficult to manage safety on construction sites [4].

Given this complexity, events like the COVID-19 pandemic present even greater challenges for a sector where health and safety management was already difficult to manage in normal times. The importance of the construction industry as an engine of global development - and its relevance in terms of the jobs it provides (especially to low-skilled workers), accounting for 18 million jobs in Europe alone - challenges the sector to reinforce its preventive health and safety measures to enable workers to continue working even during the pandemic.

In addition to these aspects, several authors have pointed to the lack of real awareness or significant training regarding health and safety issues in construction. The 
implementation of a safety culture is one of the most crucial needs in this sector. However, traditional safety training for construction workers has not had the expected effects. Lectures with little active student participation, unattractive training material and simple evaluations assessing memory are not enough. Thus, immersion in Building Information Modelling (BIM) models and training in virtual reality (VR) environments have emerged as strategies to bridge these gaps. VR allows for first-person immersion in realistic scenarios, allowing workers to interact with dynamic and risky environments [5]. The incorporation of serious game (SG) elements provides a layer of gamification, which captures workers' attention and keeps them challenged in the virtual simulations.

Although VR, virtual scenarios and SGs have proven effective in safety training in association with learning tasks, they have not succeeded in leading workers to fully internalize the message of a safety culture [6]. This goes beyond learning how to safely perform a task to extend into the realm of emotionality - that is, the worker should become truly aware of the effects of accidents and the need to behave responsibly. Under this approach, storytelling allows researchers to study the role of emotions and cultural factors as determinants of people's behaviour and a means of deepening users' awareness; users can experience the content as a protagonist who is responsible for the outcome of the story, which offers emotional meaning beyond theoretical content on safety protocols [7].

Thanks to these technologies and methods, job safety analysis technicians can quickly and easily build customised VR experiences to improve construction workers' safety awareness. Topics might include awareness of new, much more robotic-based building sites; safe ways to interact with new heavy machinery; improved sensor-based warnings for working at heights; and the use of ladders, scaffolding and ropes alongside dangerous drops, among others [8]. A last the focus of this work is social distancing adapted to the new post-COVID-19 era.

As a specific example of this versatile and fast technique, this work shows the step-bystep procedure of creating social distancing learning material for construction workers. This research shows the results of the development of a virtual experience for social distancing awareness at construction sites in the context of the COVID-19 pandemic. The tool draws on the benefits of development environments for VR experiences and their link to BIM models, which are associated with realism and immersion. In addition, scenarios for worker decision-making aligned with the generation of storytelling were developed. The aim of this research is to raise awareness of and evaluate workers' behaviour with regard to social distancing and how this new aspect can trigger the occurrence of other risky actions.

\section{RESEARCH METHODOLOGY}

The Design Science Research Methodology (DSRM) was used to guide the entire process of justification, development and testing [9]. In general, the DSRM involves four stages: (1) identifying observed problems and motivations; (2) defining the objectives of a potential solution; (3) design and development; and (4) demonstration. Fig. 1 shows a diagram of the research methodology. In the first stage, a literature review was carried out to identify the characteristics of construction safety; the impact of COVID-19; the forms of construction safety training; and how VR, SGs and storytelling improve training. The literature search was conducted on the Scopus and Web of Science databases. In the second stage, the objective of a potential solution was defined: use of VR, SG and storytelling can help safety training to raise awareness about social distancing. In the third stage, the conceptual proposal was created, and the scenario and algorithms for virtual interaction were developed. Finally, in the fourth stage, the virtual experience was implemented and initially evaluated. 


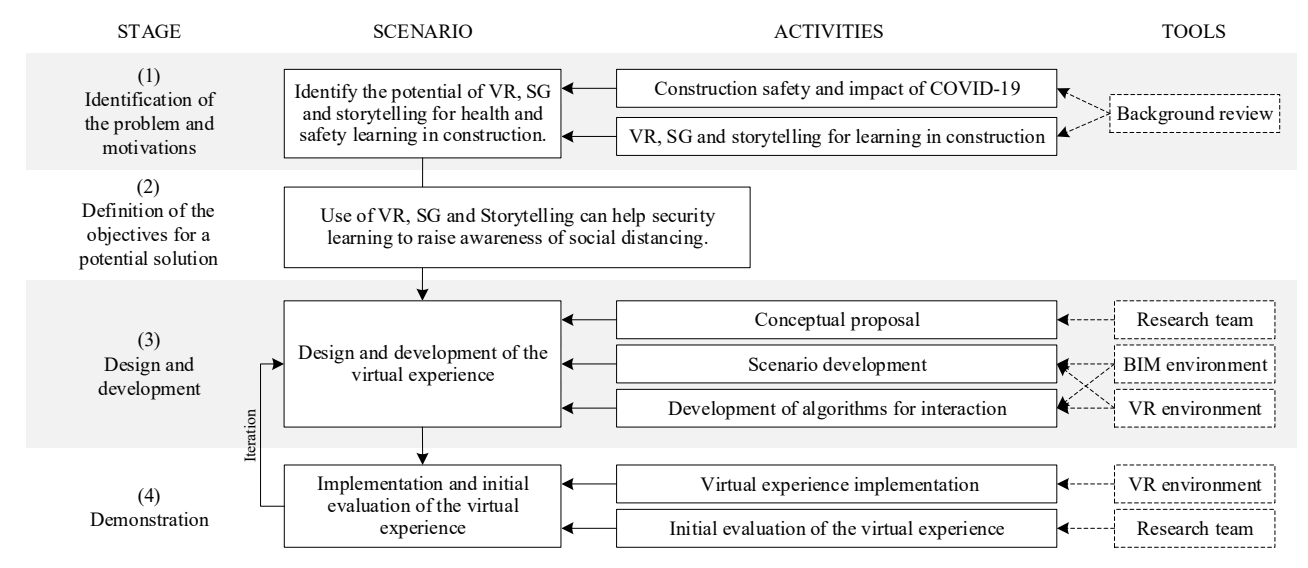

Figure 1: Research methodology.

\section{BACKGROUND}

\subsection{Health and safety in construction}

The construction sector worldwide has a high rate of occupational accidents due to high levels of risk and major safety challenges. Construction workers tend to have a higher probability of suffering an accident in the workplace because of physical overexertion, falls and trips, collisions or blows against moving objects, contact with sharp materials or being run over by construction machinery [10]. Other causes of fatal occupational accidents include heart attack and stroke, traffic accidents, entrapment and amputations, collisions with moving objects and falls [11]. In addition, the most important risk factors influencing safety performance in the construction industry are associated with poor work site setup and organisation, lack of coordination, poor communication, constant workplace changes, the different languages, cultures and educational backgrounds of the workforce, inadequate training, and the absence of a culture of prevention and safety [12].

The COVID-19 pandemic has undoubtedly impacted the construction sector. In particular, in the area of safety, the pandemic has forced the industry to incorporate new prevention measures, which are not easy to implement and control in a sector that already experienced difficulties in normal times [8]. Masking and social distancing, combined with the stressful and challenging situations that workers on construction sites already experience, increase the variables that workers must consider when carrying out their routine tasks [13].

\subsection{Health and safety training in construction}

Training in occupational health and safety in construction is considered crucial in reducing accident rates and moving towards a zero-accident culture [14]. Traditional training includes classroom lessons on theoretical concepts, manuals and digital syllabi. In this type of training, the worker plays a passive role and learning is verified through (inefficient) final questionnaires [15]. Studies show that the level of information retention decreases over time, so it is important to use tools that generate lasting learning. Traditional methods are limited to complying with the norms associated with prevention, with no intention of 
taking action or implementing training measures that lead to meaningful learning (for which real-world experience is required in addition to theoretical knowledge) [16].

\subsection{BIM, virtual reality, serious games and storytelling for training in construction safety}

In line with the trend of digitalisation in the construction sector and the increasing use of BIM, new tools for prevention and safety training have emerged [17]. BIM-based VR scenarios make it possible to generate a fully immersive, lifelike environment wherein the user experience relates to how the real world is perceived [18]. In addition, through the integration of information technology and the application of games, SGs make it possible to recreate real danger zones in a virtual environment to promote learning by configuring video games to establish a series of challenges and cognitive-behavioural changes via reward and penalty mechanisms to achieve the desired objectives [16].

Incorporating storytelling into these virtual training experiences has indicated a littleIncorporating storytelling into these virtual training experiences shows a preliminary promising series of series of characteristics that open new ways to improve the significance of the learning [19]. Storytelling refers to the art of presenting a narrative with elements that generate emotionality and transport recipients, enabling them to in a meaningful way and learn from the events being described [20]. Storytelling promotes the development of effective and selective attention, reasoning skills and cognitive skills. Its application in accident prevention has been observed in the context of road safety education. Storytelling can be used to study the role of cultural factors and emotions as determinants of people's behaviour [21]. Thus, in virtual immersive environments, the users experience content as protagonists responsible for the outcome of the story and thus receive emotional meaning beyond the theoretical content on safety protocols and standards [22].

\section{DESIGN AND DEVELOPMENT OF THE VIRTUAL EXPERIENCE}

For the development of the virtual experience, the conceptual design route considers 3 elements: (1) Learning goals, (2) VR experience goals, and (3) Decision tree linked to learning options depending on the Safety head. The learning objectives are related to: awareness of social distancing, the importance of wearing a mask, and the identification of unsafe work areas.

On the other hand, VR experience goal is to get the worker to a safe zone. In order to do so, many kind of conventional missions can be assigned, in accordance with the project requirements. The decision tree is designed to allow the user to experience the following options: the worker enters the construction site. In order to reach the defined safe zone, the worker must traverse a large part of the construction site. On the route (something to be decided by the user), the worker encounters groups of workers who must be avoided (so as not to violate social distancing). In addition, unsafe zones are placed near these workers to expose the worker to other potential risks. In other words, the worker must maintain awareness not only of people but also of unsafe typical areas of the construction site. Fig. 2 shows a simplified schematic of the virtual experience route.

Fig. 3 shows the steps to develop the virtual experience: (1) Technical project and occupational risk prevention information is obtained. With this information, (2) a BIM model of the building is designed, and its textures are improved (to obtain higher levels of realism in the virtual environment), (3) in the VR environment, physics, gravity, context elements, building site objects are added, along with programming for the user and 


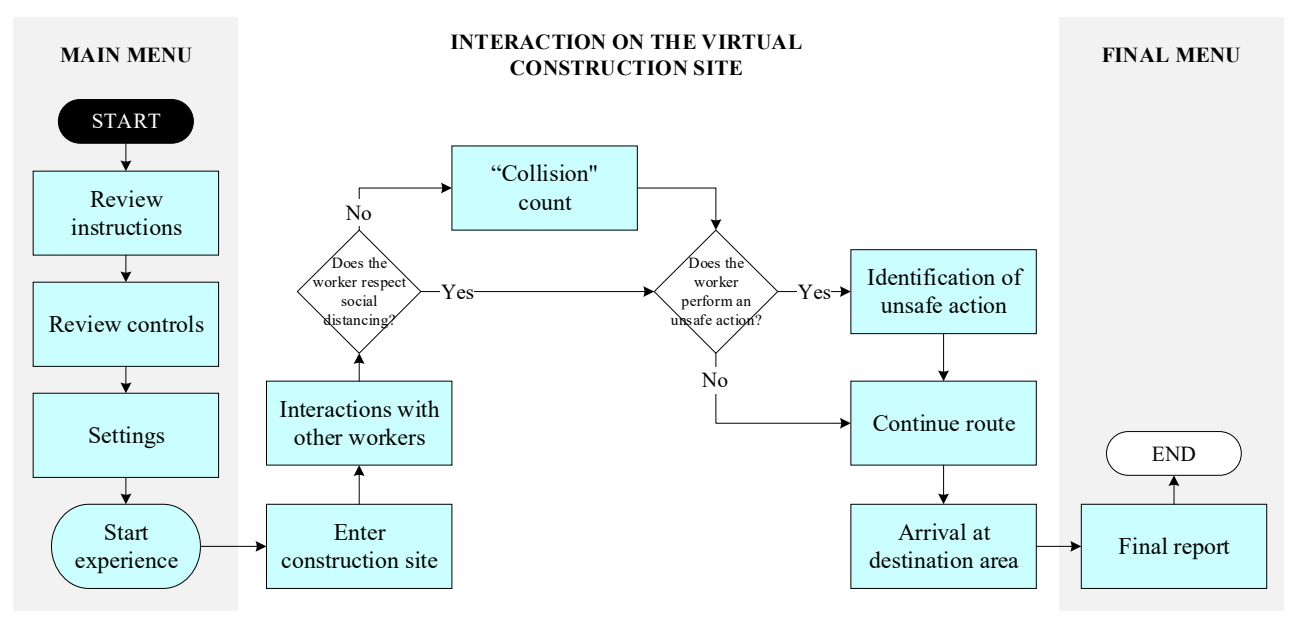

Figure 2: Diagram of virtual experience route.

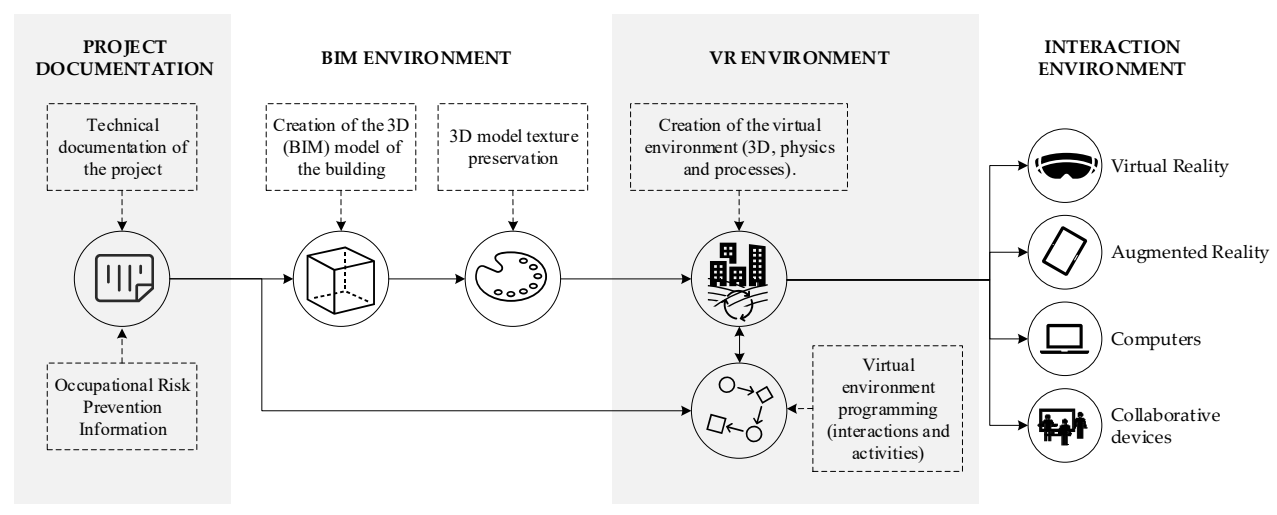

Figure 3: Tool flow for creation of virtual experience.

environment interactions. Finally, (4) the virtual experience is exported for viewing on different devices. For this particular development, the tools used included: Autodesk Revit, 3D Studio Max, Unity 3D, Visual Studio (C\#) and computer visualization of the experience.

In accordance with the training objectives, different elements were created to generate realism in the virtual environment based on the project information and security plan. Fig. 4 shows the objects in the virtual construction site: Fig. 4(a) shows the plan view of the construction site; Fig. 4(b) shows the context of the city where the construction site is located (trees, surrounding buildings, streets and cars, among others); Fig. 4(c) shows the main building (construction phase); Fig. 4(d) shows excavation areas and details of safety fences; Fig. 4(e) shows workers and material stockpile area; and Fig. 4(f) shows machinery, safety elements and secondary buildings. 


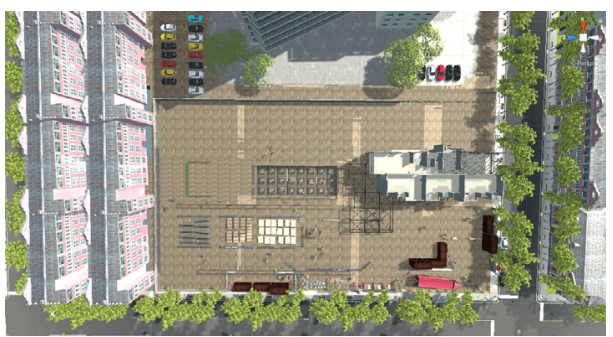

(a)

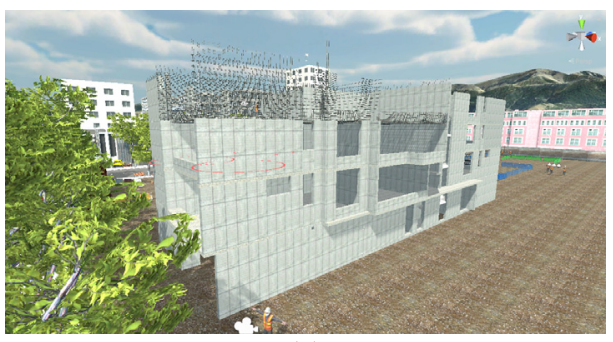

(c)

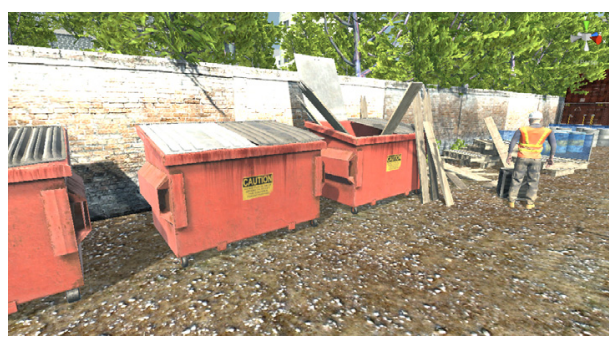

(e)

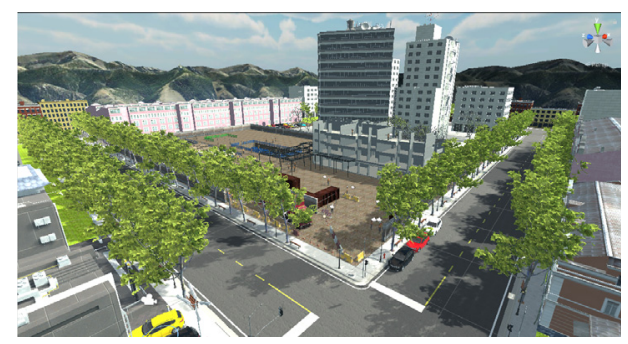

(b)

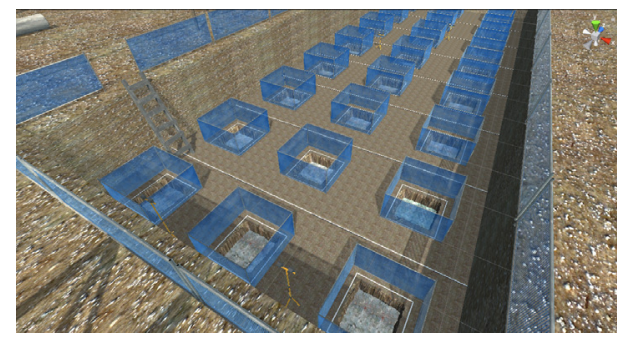

(d)

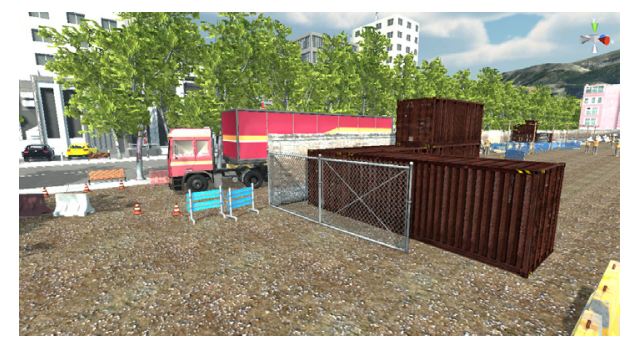

(f)

Figure 4: Virtual scenario elements. (a) General view of the construction site; (b) General site view and general elements; (c) Building view in the construction phase, imported from building information modelling environment; (d) Excavation areas; (e) Stockpile areas and other workers performing tasks; and (f) Auxiliary buildings, safety objects and machinery.

Characters were created to enable the worker (player) to interact with other (virtual) workers and to generate the social distancing aspects of the virtual experience. Based on the design of the main bot representing the player [23], elements were added to detect collisions with other workers (i.e. minimum social distance). Fig. 5 shows the collision zone and interactions between workers. Different options for prevention measures for COVID-19 may arise. One of these is the wearing of face masks: how important is the way the people wear the mask? Are we able to identify more risky situations depending on how the people wear the mask? In response to these and other questions, and to study the behaviour of workers in different cases, Fig. 6 shows the different mask-wearing options that were implemented. 


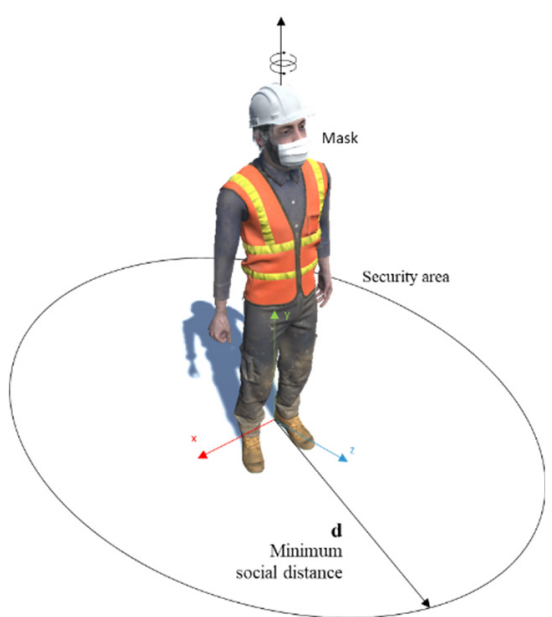

(a)

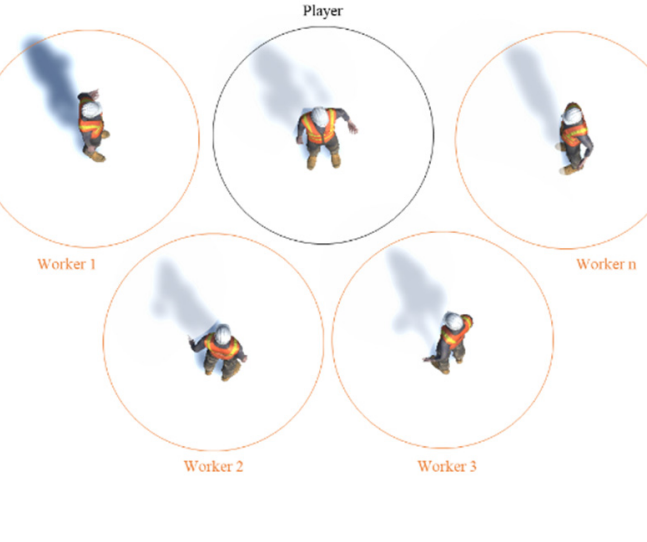

(b)

Figure 5: Safety areas for minimum social distancing. (a) Player's personal safety area; and (b) Player interaction with other workers in the virtual environment.

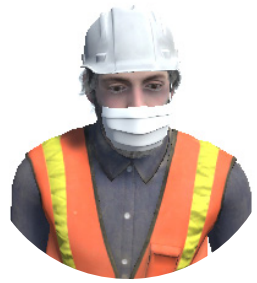

(a)

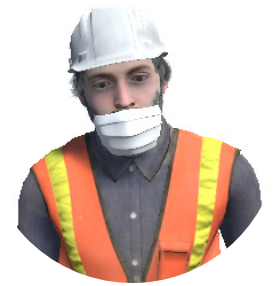

(b)

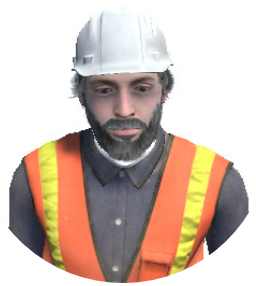

(c)

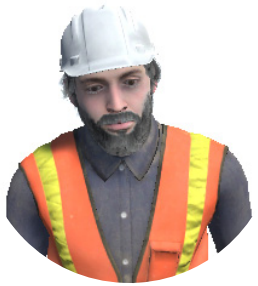

(d)

Figure 6: Options for mask wear by workers. (a) Correct wearing of mask; (b) Incorrect wearing of mask; (c) Incorrect wearing of mask; and (d) No mask.

Table 1: Algorithms developed for interaction in the virtual environment.

\begin{tabular}{|l|l|l|}
\hline$N$ & Algorithm name & Description \\
\hline 1 & Start menu & $\begin{array}{l}\text { Allows the player to access or exit the virtual experience: } \\
\text { instructions, settings, credits and start of the experience. }\end{array}$ \\
\hline 2 & Player animations & $\begin{array}{l}\text { Enables the movement and interaction of the main bot } \\
\text { associated with the player. }\end{array}$ \\
\hline 3 & $\begin{array}{l}\text { Worker } \\
\text { animations }\end{array}$ & $\begin{array}{l}\text { Enables the movement and interactions of the bots located in } \\
\text { the different parts of the construction site, both 'human-like' } \\
\text { movement and construction-related activities. }\end{array}$ \\
\hline 4 & Collision & $\begin{array}{l}\text { Establishes interaction zones for each virtual worker; detects } \\
\text { collisions between the main bot and the rest of the workers } \\
\text { (when social distance is violated). }\end{array}$ \\
\hline 5 & Collision counter & $\begin{array}{l}\text { Enables traceability of the main bot's behaviour provides } \\
\text { information on the cumulative number of collisions. }\end{array}$ \\
\hline
\end{tabular}




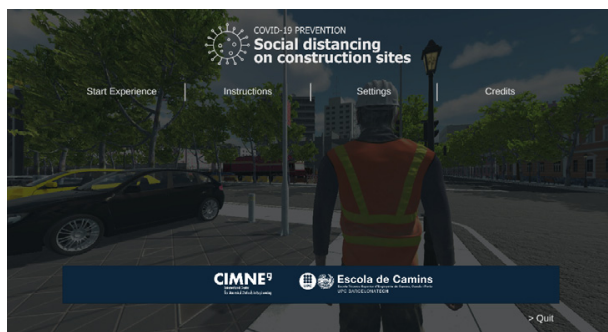

(a)

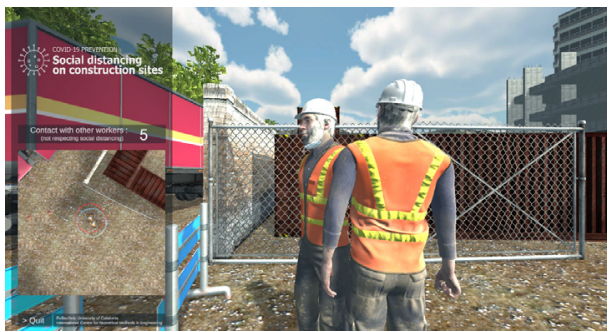

(c)

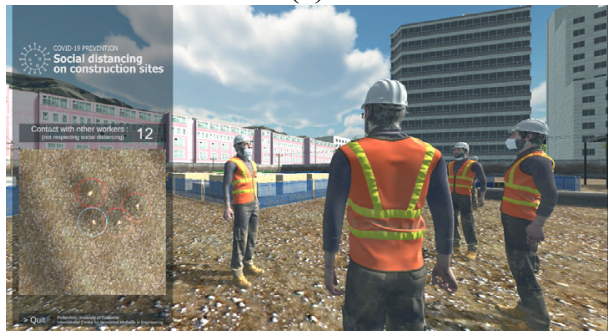

(e)

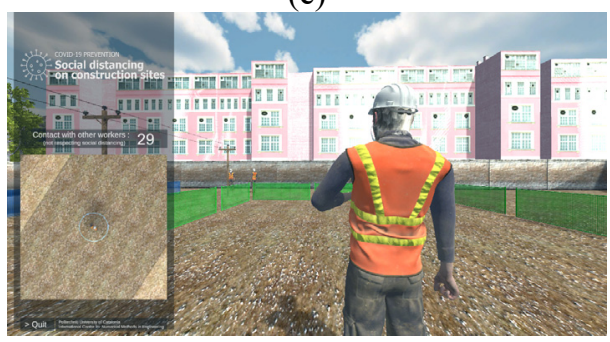

(g)

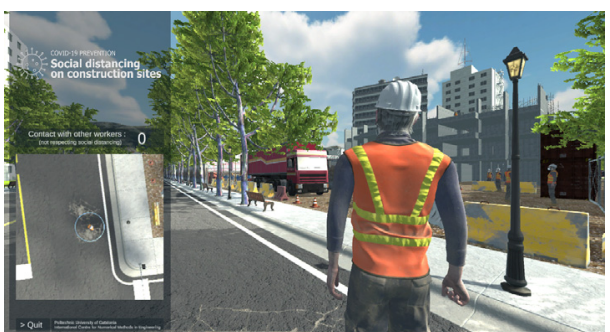

(b)

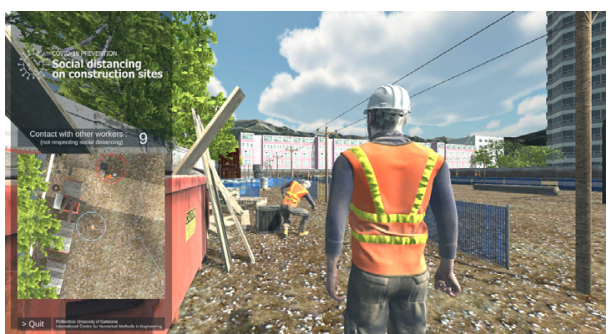

(d)

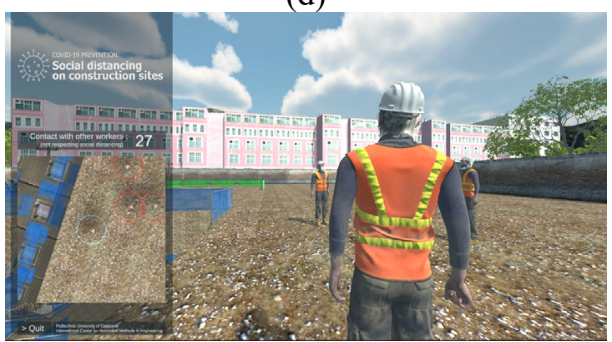

(f)

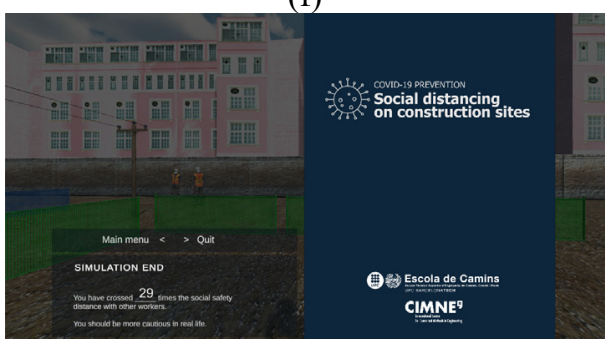

(h)

Figure 7: Scenes in virtual experience route. (a) Start menu; (b) Game start at entrance to construction site; (c) Example of virtual interaction with another worker; (d) Example of actions performed by other workers; (e) Interaction with group of workers; (f) Interaction with workers and unsafe environment; (g) Arrival at 'end zone'; and (h) Final menu.

Different algorithms were created to control the interactions of the main player, the activities of the construction site workers and the gamification aspects. Table 1 shows the five main algorithms used in the development of the virtual experience. 


\section{DEMONSTRATION OF THE VIRTUAL EXPERIENCE}

Fig. 7 shows the different 'scenes' of the route (not fixed, since the worker is free to move around the construction site). Until the defined goal area is reached, the player is able to see the start menu, the worker's trajectory and the worker's interactions with the surroundings and other workers. In Fig. 7(a), the main menu is shown, with buttons for Instructions, Configuration, Credits, and Start Experience. Fig. 7(b) shows the start of the experience and the user interface, composed of an interaction counter and a mini-map, where it is possible to see the interaction zones of the user and the workers. Fig. 7(c)-7(f) show possible interactions of the user with other workers (using the mask incorrectly, performing routine work, group worker chats, and interaction with the environment in general). Fig. $7(\mathrm{~g})$ shows the worker arriving at the safe zone, and Fig. 7(h) shows the final menu, where information on the number of interactions where the minimum social distancing is exceeded is shown.

Within the experience, which is focused on health and safety, the worker's goal is not only to maintain social distance from other workers but also to avoid risky behaviour more generally. The experience thus allows for the evaluation of how the new condition of social distancing could lead to incorrect decision-making with regard to safety matters on construction sites. For example, Fig. 8 shows part of the route taken by the player where he encounters a group of workers who must be avoided. In this context, the worker has two options: a) avoid the workers by walking to the left, which is the quickest route but would force him to walk too close to an unprotected excavation; or b) avoid them by walking to the right - a longer, but safer, route.
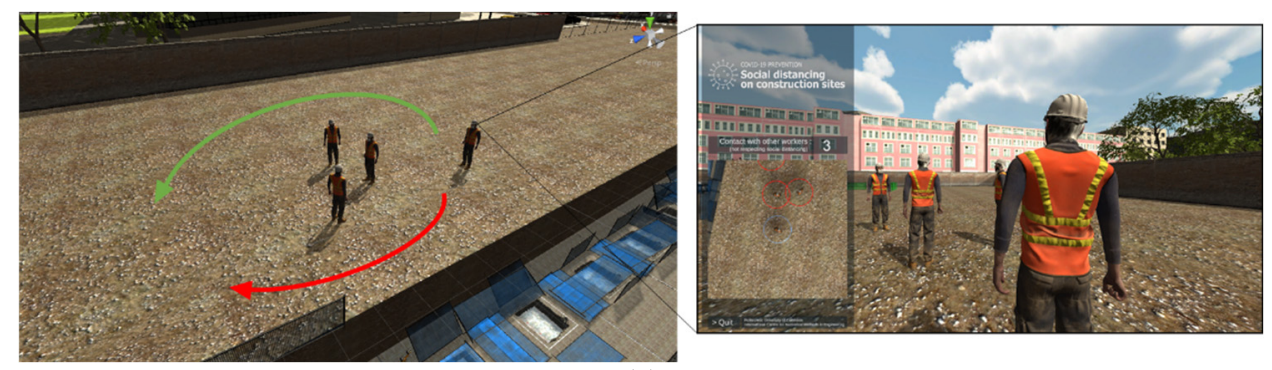

(a)

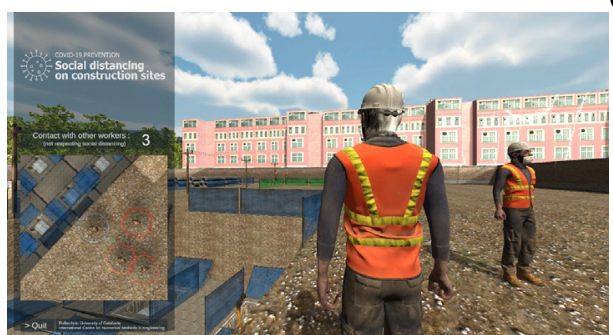

(b)

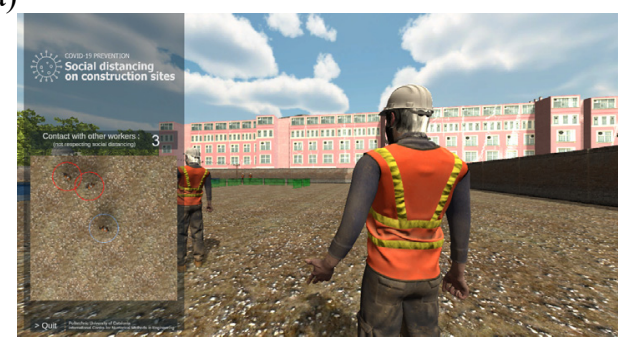

(c)

Figure 8: Example of decision-making: maintaining social distance and/or choosing a safe route. (a) Player faces best route choice decision; (b) Wrong decision (risk of fall); and (c) Correct decision (risk-free, safe area). 


\section{CONCLUSIONS}

This research successfully created a virtual experience to raise construction workers' awareness of COVID-19 prevention issues with a focus on maintaining social distancing. The developed game incorporated the integration of BIM models, the generation of realistic construction site environments and the programming of interactions and gamification aspects. It was thus demonstrated that VR can be adapted to different context, enabling the agile and simple development of customised VR experiences to raise awareness among construction workers in order to improve their safety. Thus, this research shows guides, tools, procedures, and examples for the development of virtual learning experiences.

\section{ACKNOWLEDGEMENTS}

This work has been supported by the CONICYT for its economic support to Felipe Muñoz, beneficiary of a pre-doctoral grant (CONICYT - PCHA/International Doctorate/201972200306) and by the Ministry of Science, Innovation and Universities of Spain (MICIU) through the BIMIoTICa project (RTC-2017-6454-7). The authors also acknowledge the financial support from the Spanish Ministry of Economy and Competitiveness, through the "Severo Ochoa Programme for Centres of Excellence in R\&D (CEX2018-000797-S)". The research team would like to thank Ignacio Valero (professor at the School of Civil Engineering of the Polytechnic University of Catalonia and researcher at CIMNE) for his constant support regarding health and safety issues in construction.

\section{REFERENCES}

[1] Global Construction Perspectives and Oxford Economics, A Global Forecast for the Construction Industry to 2030. Global Construction Perspectives and Oxford Economics: London, 2015.

[2] Jannadi, O.A. \& Bu-Khamsin, M.S., Safety factors considered by industrial contractors in Saudi Arabia. Building and Environment, 37(5), pp. 539-547, 2002. DOI: 10.1016/S0360-1323(01)00056-7.

[3] Ahmed, S., Causes and effects of accident at construction site: A study for the construction industry in Bangladesh. International Journal of Sustainable Construction Engineering Technology, 10(2), pp. 18-40, 2019.

DOI: 10.30880/ijscet.2 Oct. 2019.003.

[4] Khosravi, Y., Asilian-Mahabadi, H., Hajizadeh, E., Hassanzadeh-Rangi, N., Bastani, H. \& Behzadan, A.H., Factors influencing unsafe behaviors and accidents on construction sites: A review. International Journal of Occupational Safety and Ergonomics, 20(1), pp. 111-125, 2014. DOI: 10.1080/10803548.2014.11077023.

[5] Bhagwat, K., Kumar, P. \& Delhi, V.S.K., Usability of visualization platform-based safety training and assessment modules for engineering students and construction professionals. Journal of Civil Engineering Education, 147(2), 04020016, 2021. DOI: $10.1061 /($ asce $)$ ei.2643-9115.0000034.

[6] Joshi, S. et al., Implementing virtual reality technology for safety training in the precast/prestressed concrete industry. Applied Ergonomics, 90, 103286, 2021.

DOI: $10.1016 /$ j.apergo.2020.103286.

[7] Muñoz La Rivera, F., Mora Serrano, J., Delgado, C., Briceño, C., Vera, R. \& Núñez, T., Storytelling in mixed reality experiences to develop a culture of occupational risk prevention in construction, VIII Convención Internacional de la Edificación, 2020.

[8] Ogunnusi, M., Hamma-Adama, M., Salman, M. \& Kouider, T., COVID-19 pandemic: The effects and prospects in the construction industry. International Journal of Real Estate Studies, 14(2), p. 120, 2020. 
[9] Peffers, K., Tuunanen, T. \& Gengler, C., The design science research process: A model for producing and presenting information systems research. Journal of Management Information Systems, pp. 83-106, 2006.

[10] Winge, S., Albrechtsen, E. \& Mostue, B.A., Causal factors and connections in construction accidents. Safety Science, 112, pp. 130-141, 2019.

DOI: $10.1016 /$ j.ssci.2018.10.015.

[11] Yap, J.B.H. \& Lee, W.K., Analysing the underlying factors affecting safety performance in building construction. Production Planning \& Control, 2019. DOI: 10.1080/09537287.2019.1695292.

[12] Abas, N.H., Yusuf, N., Suhaini, N.A., Kariya, N., Mohammad, H. \& Hasmori, M.F., Factors affecting safety performance of construction projects: A literature review. IOP Conference Series: Materials Science and Engineering, 713(1), 2020.

DOI: $10.1088 / 1757-899 X / 713 / 1 / 012036$.

[13] Wang, Z., Liu, Z. \& Liu, J., Risk identification and responses of tunnel construction management during the COVID-19 pandemic. Advances in Civil Engineering, 2020. DOI: $10.1155 / 2020 / 6620539$.

[14] Teizer, J., Right-time vs real-time pro-active construction safety and health system architecture. Construction Innovation, 16(3), pp. 253-280, 2016.

DOI: 10.1108/CI-10-2015-0049.

[15] Yilmaz, M. \& Kanit, R., A practical tool for estimating compulsory OHS costs of residential building construction projects in Turkey. Safety Science, 101, pp. 326331, 2018. DOI: 10.1016/j.ssci.2017.09.020.

[16] Gao, Y., Gonzalez, V.A. \& Yiu, T.W., The effectiveness of traditional tools and computer-aided technologies for health and safety training in the construction sector: A systematic review. Computers \& Education, 138, pp. 101-115, 2019.

DOI: 10.1016/j.compedu.2019.05.003.

[17] Muñoz-La Rivera, F., Mora-Serrano, J., Valero, I. \& Oñate, E., Methodological Technological framework for construction 4.0. Archives of Computational Methods in Engineering, 28(2), pp. 689-711, 2021. DOI: 10.1007/s11831-020-09455-9.

[18] Kassem, M., Benomran, L. \& Teizer, J., Virtual environments for safety learning in construction and engineering: Seeking evidence and identifying gaps for future research. Visualization in Engineering, pp. 1-15, 2017.

DOI: 10.1186/s40327-017-0054-1.

[19] Pedro, A., Le, Q.T. \& Park, C.S., Framework for integrating safety into construction methods education through interactive virtual reality. Journal of Professional Issues in Engineering Education and Practice, 142(2), pp. 1-10, 2016.

DOI: 10.1061/(ASCE)EI.1943-5541.0000261.

[20] McHugh, K. \& Klockner, K., Learning lessons from rail safety storytelling: Telling safety like it is. Safety Science, 122, p. 104524, 2020.

DOI: $10.1016 /$ j.ssci.2019.104524.

[21] Oschatz, C. \& Klimmt, C., The effectiveness of narrative communication in road safety education: A moderated mediation model. Communications, 41(2), pp. 145165, 2016. DOI: 10.1515/commun-2016-0003.

[22] Cullen, E. \& Fein, A., Tell me a story: Why stories are essential to effective safety training. Report of Investigations, 2005. DOI: 10.1242/jcs.02992.

[23] Mixamo, Worker character. https://www.mixamo.com/\#/?page=1\&query=worker\& type $=$ Character. Accessed on: 10 Jan. 2021. 